\title{
O melhoramento cognitivo e a natureza humana na perspectiva transumanista
}

\author{
Cognitive enhancement and the human nature on transhumanit's \\ perspective
}

Rodrigo Pedro Mella Parmeggiani ${ }^{1}$

Kleber Bez Birolo Candiotto ${ }^{2}$

\section{Resumo}

O fenômeno da convergência tecnológica e o projeto transumanista propiciam possibilidades de melhoramento da capacidade humana, antes nunca cogitada. Porém, grandes problemáticas filosóficas são levantadas, justamente por conta dos grandes impactos éticos, epistemológicos, sociológicos causados. O problema central deste artigo refere-se a como o transumanismo concebe seu projeto de melhoramento em relação ao aspecto cognitivo e as possíveis implicações acerca da natureza humana? Como objetivo desta pesquisa buscou-se apresentar as formas de aprimoramento das capacidades cognitivas humanas sustentadas pelo projeto transumanista bem como as implicações de ordem filosófica acerca da noção de natureza humana e suas limitações. Pode-se constatar que o projeto transumanista almeja realizar modificações na natureza humana, suplantando as condições a ela impostas pelo complexo processo evolucionário. O transumanismo pretende, portanto, "domar" a casualidade e operar autonomamente modificações na condição humana, de forma a acelerar os processos de melhoria (enhancement), visando o bem estar, a qualidade de vida e de produção científico-tecnológica. $\mathrm{O}$ melhoramento cognitivo, através de uma interação cada vez mais íntima com a máquina, vislumbra para o futuro inéditas possibilidades de pensamentos, de ideias, de conhecimentos e novas formas de se relacionar com a realidade.

Palavras-chave: Transumanismo. Natureza Humana. Melhoramento cognitivo. Melhoramento Humano.

\footnotetext{
${ }^{1}$ Graduando em filosofia (bacharelado) na Escola de educação e humanidades - PUCPR. E-mail: rodrigo.parme@hotmail.com Orcid: https://orcid.org/oooo-00o3-0370-4380 2 Doutor em filosofia pela UFSCar. Professor no Programa de Pós-Graduação em Filosofia - PUCPR. E-mail: Kleber.c@pucpr.br. Orcid: https://orcid.org/oooo-0002-2000-4776
} 


\begin{abstract}
The phenomenon of technological convergence and the transhumanist project provide possibilities for improving human capacity, never before considered. However, great philosophical problems are raised, precisely because of the great ethical, epistemological and sociological impacts caused. The central problem of this article refers to how transhumanism conceives its improvement project in relation to the cognitive aspect and the possible implications on human nature? The objective of this research was to present the forms of improvement of human cognitive capacities sustained by the transhumanist project, as well as the philosophical implications about the notion of human nature and its limitations. It can be seen that the transhumanist project aims at making changes in human nature, overcoming the conditions imposed by the complex evolutionary process. Transhumanism intends, therefore, to "tame" chance and autonomously operate modifications in the human condition, in order to accelerate the processes of improvement (enhancement), aiming at well-being, quality of life, and scientifictechnological production. The cognitive enhancement, through an increasingly intimate interaction with the machine, glimpses for the future new possibilities of thoughts, ideas, knowledge and new ways of relating to reality.
\end{abstract}

Keywords: Transhumanism. Human Nature. Cognitive Enhancement. Human Enhancement.

\title{
Introdução
}

A convergência tecnológica, que é designada sob o acrônimo NBIC3, é o resultado do desenvolvimento mútuo entre nanotecnologia, biotecnologia, ciências da informação, da comunicação e ciências cognitivas. Um de seus principais resultados tem sido a artificialização de atividades de alta performance cognitiva, especialmente com o aprimoramento da inteligência artificial, que tem resultado em um cenário de supremacia das máquinas em muitas atividades que outrora eram consideradas de exclusividade humana. Dessa forma, outra perspectiva que se apresenta cada vez mais factível tem sido a integração entre o humano e a máquina, viabilizando um exponencial e inédito melhoramento das capacidades do ser humano.

O transumanismo é um movimento que "[...] promove uma abordagem interdisciplinar para entender e avaliar as oportunidades de modo a melhorar a condição humana e o organismo humano frente às tecnologias em andamento"

3 Nanotecnologia, Biotecnologia, Informática (big data, internet das coisas) e Cognitivismo (inteligência artificial e robótica). 
(BOSTROM, 2005, p. 3, tradução nossa)4. As formas de aprimoramento que o transumanismo ressalta vão desde a extensão de tempo da vida humana, erradicação de doenças, eliminação do sofrimento desnecessário, aumento das capacidades intelectuais, físicas e emocionais, até a colonização espacial, a possibilidade de criar máquinas superinteligentes, e outros desenvolvimentos que poderiam modificar de forma significativa a condição existencial dos indivíduos (BOSTROM, 2005). Além de se constituir como um movimento intelectual e cultural, o transumanismo se caracteriza por buscar o aprimoramento da qualidade de vida e seu prolongamento. $\mathrm{O}$ aprimoramento ou melhoria (enhancement) é um objetivo visado pelo transumanismo que busca se utilizar da tecnologia em progresso, tais como, as células-tronco, clonagem reprodutiva, hibridação homem/máquina, engenharia genética e manipulações germinais, para poder modificar nossa espécie de modo irreversível, no intuito de melhorar a condição humana.

Além disso, o transumanismo propõe um alargamento da perspectiva tradicional da medicina como ideal tão somente terapêutico. O paradigma da medicina associado exclusivamente ao método reparativo seria considerado pelo transumanismo como insuficiente, demandando uma ampliação do uso de certas técnicas em vista da melhoria /aumento. Não se trataria apenas de operar uma reparação no ser humano, mas sim aumentá-lo (improvement) ou melhorá-lo (enhancement) (FERRY, 2018).

Em contraposição as ideias apresentadas, surgem críticas por parte dos chamados bioconservadores. Em termos gerais, os bioconservadores tendem a se opor ao uso das biotecnologias em vista da modificação da natureza humana, pois em suas concepções, tais tecnologias comprometeriam a dignidade humana e potencializariam fatores desumanizantes (PESSINI, 2006). De acordo com Vilaça e Dias "De um ponto de vista ou de outro, os bioconservadores amparam-se no conceito de natureza humana, religioso ou secular, para argumentar contra as biotecnologias com fins de seleção e melhoramento humanos" (VILAÇA; DIAS, 2014, p. 354).

A temeridade bioconservadora recai sobre o problema da instrumentalização

\footnotetext{
4 It promotes an interdisciplinary approach to understanding and evaluating the opportunities for enhancing the human condition and the human organism opened up by the advancement of technology.
} 
da biologia humana, na medida que compreendem que isso poderia solapar a humanidade e os seus valores fundamentais (VILAÇA; DIAS, 2014). Na perspectiva bioconservadora, como é o caso de Francis Fukuyama, não respeitar a natureza humana, querer modifica-la, seria "[...] simplesmente destruir os fundamentos naturais da ética" (FERRY, 2018, p. 45). Assim, os princípios éticos teriam sua fundamentação a partir da natureza humana, estando os direitos naturais do homem ligados a sua constituição biológica. Essa é a problemática que converge para a temática geral deste trabalho. $\mathrm{O}$ aprimoramento cognitivo e mudanças genéticas poderiam acarretar uma mudança irreversível na natureza humana? Se sim, isso significaria algo positivo ou negativo?

O otimismo tecnocientífico quanto aos benefícios do transumanismo é resultante de uma confiança no progresso comparável a que animava os intelectuais na época do iluminismo. Por isso, semelhante ao projeto iluminista, o projeto transumanista visa superar as limitações humanas dadas pela sua própria natureza. O presente trabalho, portanto, se estrutura a partir do seguinte problema: como o transumanismo concebe seu projeto de melhoramento em relação ao aspecto cognitivo e as possíveis implicações acerca da natureza humana?

\section{O melhoramento cognitivo}

Os melhoramentos firmados na proposta transumanista tem em vista potencializar as qualidades físico-biológicas (e por vezes com pretensões de melhoria moral) dos humanos. O controle do homem sobre a própria natureza que o constitui seria a idealização do projeto transumanista em vista de atingir o estágio pós-humano, estágio caracterizado por um salto das condições humanas atuais para outras completamente inéditas. Dentre os vários tipos de aprimoramento possíveis e vislumbrados para o futuro, os transumanistas defendem o uso consciente da tecnologia em progresso para o aprimoramento da cognição. Nas palavras de Bostrom e Sandberg:

O aprimoramento cognitivo pode ser definido como a amplificação ou extensão das principais capacidades da mente através do aprimoramento ou aumento dos sistemas de processamento de informação internos ou externos. A cognição, por sua vez, pode ser definida como os processos que um organismo usa para organizar as informações. Isso inclui a aquisição de informações (percepção), seleção (atenção), representação (compreensão) e retenção (memória) de informações, e o uso para orientar 
o comportamento (raciocínio e coordenação das saídas do motor). Intervenções para melhorar a função cognitiva podem ser direcionadas a qualquer uma dessas faculdades centrais (BOSTROM; SANDBERG, 2006, p. 201)5.

Para a compreensão do que compreende o melhoramento cognitivo, primeiramente deve-se compreender o que significa a cognição propriamente dita. De acordo com Bostrom e Sandberg (2006), em seu artigo intitulado Converging Cognitive Enhancement, a cognição poderia ser definida como:

[...] os processos que um organismo usa para organizar as informações. Isso inclui a aquisição de informações (percepção), seleção (atenção), representação (compreensão) e retenção (memória) de informações, e o uso para orientar o comportamento (raciocínio e coordenação das saídas motoras). Intervenções para melhorar a função cognitiva podem ser direcionadas a qualquer uma dessas faculdades centrais (BOSTROM; SANDBERG, 2006, p. 201, tradução nossa) ${ }^{6}$.

Sandberg e seu artigo denominado Cognition Enhancement: Upgrading the Brain, define o melhoramento (enhancement) como: “[...] uma intervenção que melhora um subsistema de uma forma que não seja reparar algo que está defeituoso ou remediar uma disfunção específica” (SANDBERG, 2011, p. 71, tradução nossa)7. Assim, uma pessoa melhorada/aprimorada cognitivamente "[...] é alguém que é beneficiada por uma intervenção que melhora o desempenho de algum subsistema cognitivo sem corrigir algo específico, patologia identificável ou disfunção desse subsistema." (SANDBERG, 2011, p. 71, tradução nossa). ${ }^{8}$ Bostrom e Sandberg definem o melhoramento da cognição como "[...] a amplificação ou extensão das capacidades centrais da mente através da melhoria ou aumento de sistemas de

\footnotetext{
5 Cognitive enhancement may be defined as the amplification or extension of core capacities of the mind through improvement or augmentation of internal or external information processing systems. Cognition in turn can be defined as the processes an organism uses to organize information. This includes both the acquisition of information (perception), selecting (attention), representing (understanding), and retaining (memory) information, and using it to guide behavior (reasoning and coordination of motor outputs). Interventions to improve cognitive function may be directed at any one of these core faculties.

${ }^{6}[\ldots]$ the processes an organism uses to organize information. This includes both the acquisition of information (perception), selecting (attention), representing (understanding), and retaining (memory) information, and using it to guide behavior (reasoning and coordination of motor outputs). Interventions to improve cognitive function may be directed at any one of these core faculties.

$7[\ldots]$ it is an intervention that improves a subsystem in some way other than repairing something that is broken or remedying a specific dysfunction."

8 [...] is somebody who has benefited from an intervention that improves the performance of some cognitive subsystem without correcting some specific, identifiable pathology or dysfunction of that subsystem.
} 
processamento de informação internos ou externos" (BOSTROM; SANDBERG, 2006, p. 201, tradução nossa)9.

Acerca do que caracteriza o melhoramento cognitivo, Bostrom explicita que o desenvolvimento neurológico pode ser alcançado por vias ditas naturais como "[..] melhorias na nutrição materna e infantil, remoção de chumbo e outros poluentes neuro-tóxicos do meio ambiente, erradicação de parasitas, incentivo a níveis adequados de sono e exercício[...]" (BOSTROM, 2018, p. 68). As melhorias poderiam acontecer por tais alternativas, porém, os resultados obtidos seriam bem modestos e vagarosos. Por isso "[...] a melhoria da biomedicina poderia permitir impulsos maiores. Atualmente já existem drogas que supostamente melhoram a memória, a concentração e a energia mental em pelo menos alguns aspectos." (BOSTROM, 2018, p. 68).

Os tipos de melhoramentos cognitivos variam e cada tipo detém características específicas. O que se pode citar de início são as práticas educativas e de treinamento mental em que "[...] o objetivo geralmente é não apenas transmitir habilidades ou informações específicas, mas também melhorar as faculdades mentais gerais, como concentração, memória e pensamento crítico" (SANDBERG, 2011. p. 72, tradução nossa) ${ }^{10}$. Sandberg apresenta ainda a distinção de melhoramento cognitivos convencionais, como os citados acima, e os nãoconvencionais, "[...] como aqueles que envolvem drogas nootrópicas criadas deliberadamente, terapia genética ou implantes neurais, são quase todos considerados experimentais no presente momento" (SANDBERG, 2011, p. 72, tradução nossa) ${ }^{11}$. São esses últimos, os não-convencionais, que mais interessam ao debate filosófico transumanista.

A biotecnologia farmacêutica é um tipo de aprimoramento acessível e bastante utilizado. Substâncias estimulantes como a nicotina e a cafeína são muito usadas para melhorar o desempenho da cognição, bem como as substâncias estimulantes que abrangem os hormônios, nutrientes, etc. Sobre o melhoramento por fármacos, os autores Bostrom e Sandberg expressam o seguinte:

\footnotetext{
9 [...] the amplification or extension of core capacities of the mind through improvement or augmentation of internal or external information processing systems.

${ }^{10}[\ldots .$.$] where the goal is often not only to impart specific skills or information, but also to improve$ general mental faculties such as concentration, memory, and critical thinking.

${ }^{11}[. .$.$] such as ones involving deliberately created nootropic drugs, gene therapy, or neural implants,$ are nearly all to be regarded as experimental at the present time.
} 
[...] o aprimoramento farmacológico é possível aqui e agora, embora as melhorias na capacidade tendam a ser uma melhoria modesta de 10 a $20 \%$ nos resultados dos testes. Quanto a toda farmacologia, existem grandes variações interindividuais. O uso otimizado de medicamentos pode incluir testes dos níveis de neuromodulador para ver onde estão os pontos de ajuste do cérebro, testes farmacogenômicos para descobrir como eles são metabolizados e testes neuropsicológicos para verificar quais níveis produzem desempenho máximo (BOSTROM; SANDBERG, 2006, p. 203, tradução nossa) ${ }^{12}$.

Outro tipo de melhoramento apresentado por Bostrom e Sandberg é a estimulação magnética transcraniana (EMT), ${ }^{13}$ que “[...] estimula os neurônios no córtex cerebral, alterando o campo magnético induzido por uma bobina presa à cabeça. Pode aumentar ou diminuir a excitabilidade do córtex, alterando assim seu nível de plasticidade” (BOSTROM; SANDBERG, 2006, p. 206, tradução nossa) ${ }^{14}$. Segundo os autores o EMT foi considerado promissor em determinadas tarefas como por exemplo: tarefas motoras, aprendizado motor, tarefas de coordenação visomotora, memória de trabalho, toque na sequência dos dedos, classificação e até consolidação de memória declarativa durante o sono (BOSTROM; SANDBERG, 2006).

Um outro tipo de melhoramento cognitivo apresentado por Bostrom e Sandberg é o treino mental. Treinos mentais normalmente trabalham com a memória, ou seja, são estratégias para aumentar a capacidade de memorizar informações. Como exemplo disso, pode-se citar o palácio da memória, ou método de loci (BOSTROM; SANDBERG, 2006). Os autores ainda ressaltam que “[...] existe uma vasta gama de técnicas mentais que supostamente aumentam várias habilidades, como treinamento de criatividade, métodos de leitura rápida e mapas mentais” (BOSTROM; SANDBERG, 2006, p. 211, tradução nossa) ${ }^{15}$.

Existe uma vasta gama de tecnologia da informação que possuem um papel de melhoramento cognitivo. Segundo os autores "O hardware externo já é usado

\footnotetext{
${ }_{12}$ [...] pharmacological enhancement is possible here and now, although the improvements in ability tend to be a modest $10-20 \%$ improvement of test scores. As for all pharmacology, there are great interindividual variations. Using enhancer drugs optimally might include tests of neuromodulator levels to see where the brain setpoints are, pharmacogenomic tests to find how they are metabolized and neuropsychological tests to check what levels produce maximum performance. Such fine-tuning is expensive and cumbersome unless it can be automated.

13 Transcranial Magnetic Stimulation (TMS).

14 [..]stimulates neurons in the cerebral cortex by a changing magnetic field induced from a coil held to the head. It can increase or decrease the excitability of the cortex, thereby changing its level of plasticity.

${ }_{15}$ [...] exists a vast array of mental techniques alleged to boost various skills, such as creativity training, speed reading methods, and mind-maps.
} 
para aprimoramento cognitivo, seja de caneta e papel ou software de computador, como organizadores pessoais.” (BOSTROM; SANDBERG, 2006, p. 211, tradução nossa) ${ }^{16}$. E que "Não há área cognitiva onde não exista amplificação externa de hardware e software" (BOSTROM; SANDBERG, 2006, p. 211, tradução nossa) ${ }^{17}$. Segundo os autores:

O que há de novo é o crescente interesse em criar vínculos íntimos entre os sistemas externos e o usuário humano por meio de uma melhor interação. O software se torna menos uma ferramenta externa e mais um mediador "exoself". Isso pode ser alcançado por meio da mediação [...] como computadores portáteis ou realidade virtual, ou através de ambientes inteligentes, onde as capacidades dos objetos no ambiente são estendidas (BOSTROM; SANDBERG, 2006, p. 211, tradução nossa) ${ }^{18}$.

Nesse sentido, Sandberg no artigo Cognition enhancement: Upgrading the brain expõe o seguinte:

Dada a disponibilidade de suporte de memória externa, da gravação a computadores vestíveis, é provável que a forma crucial de demanda de memória para humanos no futuro seja cada vez mais a capacidade de vincular informações a conceitos, associações e habilidades utilizáveis, em vez da capacidade para memorizar grandes quantidades de dados brutos. As funções de armazenamento e recuperação muitas vezes podem ser descarregadas do cérebro, enquanto o conhecimento, estratégias e associações que vinculam os dados à cognição hábil ainda não podem ser terceirizados para computadores. Isso valorizará algumas habilidades cognitivas (SANDBERG, 2011, p. 78, tradução nossa) ${ }^{19}$.

No contexto do melhoramento cognitivo destaca-se também o denominado melhoramento coletivo (collective enhancement). Esse seria um tipo de melhoramento que ocorre "[...]aumentando o desempenho dos membros individuais do grupo (melhorando o desempenho geral), melhorando sua capacidade de coordenar (possibilitando grupos maiores) ou melhorando as

\footnotetext{
${ }^{16}$ External hardware is of course already used for cognitive enhancement, be it pen and paper or computer software like personal organizers.

${ }^{17}$ There is practically no cognitive area where there does not exist external hardware or software amplification.

${ }^{18}$ What is new is the growing interest in creating intimate links between the external systems and the human user through better interaction. The software becomes less an external tool and more of a mediating "exoself." This can be achieved through mediation, embedding the human within an augmenting "shell," such as wearable computers or virtual reality, or through smart environments, where capabilities of objects in the environment are extended.

19 Given the availability of external memory support, from writing to wearable computers, it is likely that the crucial form of memory demand on humans in the future will increasingly be the ability to link information into usable concepts, associations, and skills rather than the ability to memorize large amounts of raw data. Storage and retrieval functions can often be offloaded from the brain, while the knowledge, strategies, and associations linking the data to skilled cognition cannot so far be outsourced to computers. This will put a premium on some cognitive abilities.
} 
sinergias geradas por ter várias competências.” (BOSTROM; SANDBERG, 2006, p. 213, tradução nossa) ${ }^{20}$. Assim, segundo dos autores até mesmo a Internet e o e-mail (e por extensão todos os outros mecanismos que se estruturam de forma similar), podem ser considerados como grandes softwares de aprimoramento da cognição humana. Pois, através deles:

[...] a inteligência distribuída de grandes grupos pode ser compartilhada e aproveitada para fins específicos. Os sistemas conectados permitem que muitas pessoas colaborem na construção de conhecimento e soluções compartilhados: quanto mais indivíduos se conectam, mais poderoso o sistema se torna. A informação não é apenas armazenada nos próprios documentos, mas em suas inter-relações (BOSTROM; SANDBERG, 2006, p. 213, tradução nossa) ${ }^{21}$.

Portanto, quanto mais interação de indivíduos na esfera virtual, mais poder o sistema tem, e dessa forma “[...] a informação não é apenas armazenada nos próprios documentos, mas em suas interrelações” (BOSTROM; SANDBERG, 2006, p. 213) $)^{22}$.

Bostrom expõe que é pouco provável, tanto da perspectiva neurológica quanto da evolucionária, a chance de aumentar bruscamente a inteligência de um humano saudável através de medicação ou elemento químico no cérebro. Segundo ele:

O funcionamento cognitivo do cérebro humano depende de um delicado equilíbrio entre diversos fatores, especialmente durante os estágios críticos do desenvolvimento embrionário - e é muito mais provável que, para ser melhorada, essa estrutura auto-organizável precise ser cuidadosamente balanceada, sintonizada e cultivada em vez de simplesmente receber a introdução de uma poção química estranha (BOSTROM, 2018, p. 68).

Por conseguinte, Bostrom expõe que a manipulação genética é o que poderá fornecer aparato mais eficaz e poderoso do que a psicofarmacologia (BOSTROM, 2018). Dessa forma, “[...] a modificação genética tornaria o indivíduo independente de um suprimento externo de medicamento [...]" (BOSTROM; SANDBERG, 2006, p. 207, tradução nossa) 23 . Toda via, “[...] o potencial máximo de uma máquina

\footnotetext{
${ }^{20}[\ldots]$ increasing the performance of individual group members (improving overall performance), improve their ability to coordinate (enabling larger groups), or improve the synergies generated by having multiple competencies.

${ }^{21}[\ldots]$ the distributed intelligence of large groups can be shared and harnessed for particular purposes. Connected systems allow many people to collaborate in the construction of shared knowledge and solutions: the more individuals that connect, the more powerful the system becomes. The information is not just stored in the documents themselves but in their interrelations.

$22[\ldots]$ the information is not just stored in the documents themselves but in their interrelations.
} 
inteligente é, claramente, muito superior ao da inteligência orgânica" (BOSTROM, 2018, p. 76). As melhorias que podem se efetuar na capacidade biológica da cognição teriam consequências importantes, tais como proporcionar maior velocidade e produção de qualidade nas áreas da ciência e tecnologia, incluindo os desenvolvimentos na área de amplificação de inteligência biológica e da inteligência artificial (BOSTROM, 2018).

Sobre se deve-se ou não realizar o melhoramento cognitivo, Bostrom e Sandberg argumentam que mesmo que as evidências apontem que a alta inteligência não necessariamente promova felicidade aos indivíduos, é certo que a baixa inteligência acarreta inúmeros eventos negativos, como por exemplo, o aumento do risco de acidentes e a baixa renda, "[...] enquanto que a maior inteligência promove a saúde e riqueza" (BOSTROM; SANDBERG, 2006, p. 201, tradução nossa) ${ }^{24}$.

\section{A concepção de natureza humana no transumanismo}

As problemáticas envolvendo a temática do transumanismo, em especial o melhoramento humano, tendem a desembocar na querela filosófica acerca da natureza humana. Este conceito filosófico remonta a Aristóteles que afirmava que a natureza humana é concebida como "[...] um conjunto de características que são comuns a todos os seres humanos e que distinguem os humanos de outros tipos de seres" (BUCHANAN, 2009, p. 142, tradução nossa)25. Dessa forma, instituiu-se uma concepção essencialista que perpassa ao longo de toda a tradição filosófica. As problematizações acerca desse conceito geralmente são associadas a questões de âmbito moral e menos no sentido biológico. Porém, com o desenvolvimento da tecnologia e o desabrochar da possibilidade de uma modificação real na constituição físico-biológica do humano, o conceito de natureza humana se torna um importante objeto de investigação filosófica.

Nilsen aborda a problemática que permeia o conceito de natureza: "O problema, no entanto, é que 'natureza' e / ou 'natureza humana' é referida como se fosse um conceito claramente definido, e não um conceito nebuloso que abraça uma

\footnotetext{
23 [...] genetic modification would make the individual independent of an external drug supply [...]. $24[\ldots]$ while higher intelligence promotes health and wealth.

25 [...] a set of characteristics that are common to all humans and that distinguish humans from other kinds of beings.
} 
série de significados" (NIELSEN, 2011, p. 22, tradução nossa) ${ }^{26}$. Assim, o que se percebe no debate acerca da definição de natureza humana na querela envolvendo o transumanismo, é que, por vezes, o conceito acaba sendo instrumentalizado à maneira do autor (por vezes também do interlocutor) de acordo com suas convicções. Nielsen resume a questão no seguinte sentido:

\begin{abstract}
A maneira como a "natureza" e a "natureza humana" são interpretadas em relação às novas tecnologias emergentes depende muito da tradição - as formas como a natureza foi interpretada na cultura, história e religião. A ideia de um bem intrínseco na "natureza" e no "natural" pode, é claro, basear-se em motivos religiosos específicos, mas também está embutida em outras ideias (NIELSEN, 2011, p. 24, tradução nossa) ${ }^{27}$.
\end{abstract}

Por conta dessa característica que assume o debate em torno do conceito de natureza humana o cenário que se desenrolam as discussões filosóficas dividiu-se em dois, entre os que concebem as modificações/melhoramentos como algo que “[...] solaparia, desde a origem (genos=genética), a liberdade e autonomia humanas" (VILAÇA; DIAS, 2014, p. 355), e os que defendem que "[...] levaria o humano ao estágio pós-humano, sendo capaz de promover e elevar essas capacidades, dentre os benefícios citados alhures" (VILAÇA; DIAS, 2014, p. 355). Como apresentado na introdução desse artigo, os indivíduos que se põe contra essas intervenções que visam o melhoramento são conceituados como bioconservadores. Esses tendem a entender a natureza humana como algo bem definido, estático, e que detém uma essência (metafísica-transcendental ou físico-biológica). Bioprogressistas fazem parte do grupo de indivíduos convictos de que as formas de aprimoramento devem ser encorajadas, que percebem a natureza humana como algo em constante modificação no processo evolucionário e que caberia ao próprio homem direcionar essas modificações para que forneçam resultados mais eficazes, imediatos e benéficos.

De acordo com Nick Bostrom natureza humana é concebida pelo transumanismo como um "trabalho-em-desenvolvimento" 28 uma espécie de "começo inacabado" 29 que pode ser remodelado de forma progressiva, mostrando

${ }^{26}$ The problem, however, is that "nature" and/or "human nature" is referred to as if it were a clearly defined concept, and not a nebulous concept that embraces a host of meanings.

${ }_{27}$ The way "nature" and "human nature" is interpreted in relation to the emerging new technologies is highly dependent on tradition - the ways nature has been interpreted in culture, history, and religion. The idea of an intrinsic good in "nature" and the "natural" might, of course, be based on specific religious grounds, but it is also embedded in other ideas.

${ }_{28}^{28}$ work-in-progress

29 half-baked beginning 
que o status atual da natureza humana não precisa ser o ponto final (BOSTROM, 2005). O projeto transumanista, segundo ele, espera que o ser humano possa vir a tornar-se um pós-humano com capacidades mais desenvolvidas das que atualmente os seres humanos detêm (BOSTROM, 2005).

Norman Daniels apresenta sua concepção acerca da natureza humana e se fundamenta em três conceitos para defini-la: “[...] é um conceito que se aplica às populações, é disposicional e é seletivo" (DANIELS, 2009 p. 34, tradução nossa)30. Ao que Bostrom e Savulescu esclarecem as afirmações de Daniels:

A natureza humana, diz Daniels, é um conceito de população, seletiva e disposicional. É disposicional no sentido de que a mesma natureza humana se manifestará como fenótipos muito diferentes, dependendo do ambiente em que é colocada. É um conceito populacional no sentido de que, para caracterizar a natureza humana, devemos agregar variações individuais. E é seletivo no sentido de que algumas características (por exemplo, racionalidade) costumam ser mais centrais à natureza humana do que outras (por exemplo, pelos nasais). Por esse motivo, não podemos modificar a natureza humana, a menos que ajamos em nível populacional. Além disso, a intervenção deve afetar características centrais dessa natureza. Para Daniels, isso sugere que "as intervenções genéticas têm menos probabilidade de serem ameaças do que as intervenções ambientais que minam nossas capacidades ou natureza humanas (BOSTROM; SAVULESCU, 2009, p. 4, tradução nossa)31.

Na perspectiva de Daniels, uma alteração específica, por si só, no genótipo do ser humano não seria suficiente para determinar o que é a natureza humana propriamente dita. A natureza de determinada espécie dependeria exclusivamente do ambiente no qual está inserida. Além disso, o genótipo que constitui o ser humano é consequência do acaso, de milhares de anos de sucessivas alterações, sendo o meio ambiente nesse processo um fator decisivo e importante (VILAÇA; DIAS, 2014).

Vilaça e Dias, ao explicarem a concepção de natureza humana de Savulescu e Persson, apresentam-na como algo que não dispõe de uma característica boa $a$ priori, nem essencialista ou metafísica-transcendental, mas sim, meramente

\footnotetext{
$3^{30}[\ldots]$ it is a concept that applies to populations, it is dispositional, and it is selective.

${ }^{31}$ Human nature, says Daniels, is a dispositional selective population concept. It is dispositional in the sense that the same human nature will manifest as very different phenotypes depending on the environment in which it is placed. It is a population concept in the sense that to characterize human nature we must aggregate across individual variation. And it is selective in the sense that some traits (e.g., rationality) are often claimed to be more central to human nature than others (e.g., nasal hair). On this account, we cannot modify human nature unless we act on a population level. Furthermore, the intervention must affect traits central to that nature. To Daniels, this suggests that "genetic interventions are less likely to be threats than environmental interventions that undermine our human capabilities or nature.
} 
contingencial. Segundo eles, o termo humano é uma classificação biológica, quer dizer antes de tudo que tal indivíduo faz parte da espécie Homo Sapiens (VILAÇA; DIAS, 2014). Portanto, para Vilaça e Dias explicando Savulescu e Persson:

Para os autores, qualquer que seja a definição de humano, não há nenhuma virtude especial (maior valor) em fazer parte da espécie humana, pois "pertencer à espécie humana" é uma mera contingência, podendo acarretar até mesmo certos prejuízos (VILAÇA; DIAS, 2014, p. 351).

A questão do melhoramento, além de implicar problemas como o da natureza humana, acaba por problematizar outra questão, a saber: quais melhoramentos realizar? E disso decorre que saber quais melhoramentos realizar implica em determinar quais são as limitações humanas em seu estado dito natural.

Bostrom esclarece que "O alcance de pensamentos, sentimentos, experiências e atividades acessíveis aos organismos humanos presumivelmente constitui apenas uma pequena parte do que é possível" (BOSTROM, 2005, p. 4, tradução nossa) ${ }^{32}$. Ou ainda, em seu artigo Human Genetic Enhancements, afirma que: "Não há razão para pensar que o modo de ser humano esteja mais livre de limitações impostas por nossa natureza biológica do que os modos de ser de outros animais" (BOSTROM, 2003, p. 494, tradução nossa) 33 . Dessa forma, Bostrom define a natureza humana como algo limitado, propenso a melhorias ou desenvolvimento de novas potencialidades. Segundo ele, "Nosso próprio modo atual de ser, portanto, abrange apenas um subespaço minúsculo do que é possível ou permitido pelas restrições físicas do universo" (BOSTROM, 2003, p. 494, tradução nossa) 34 .

Bostrom se propõe a pensar o corpo e sua funcionalidade como uma área constitutiva da natureza humana que pode ter seu limite expandido. De fato, os seres humanos já empreendem um processo que busca expandir o estado normal do corpo e suas funcionalidades, "Nós aprimoramos nosso sistema imunológico natural recebendo vacinas e podemos imaginar novos aprimoramentos em nossos corpos que nos protegeriam de doenças ou nos ajudariam a moldar nossos corpos

\footnotetext{
${ }^{32}$ The range of thoughts, feelings, experiences, and activities accessible to human organisms presumably constitute only a tiny part of what is possible.

33 There is no reason to think that the human mode of being is any more free of limitations imposed by our biological nature than are the modes of being of other animals.

34 Our own current mode of being, therefore, spans but a minute subspace of what is possible or permitted by the physical constraints of the universe.
} 
de acordo com nossos desejos" (BOSTROM, 2005, p. 6, tradução nossa)35. O autor ainda expõe a possibilidade de interação entre mente e máquina, num contexto em que se poderia enviar uma determinada mente humana a um computador "[...] replicando in silico ${ }^{36}$ os processos computacionais detalhados que normalmente ocorreriam em um cérebro humano específico" (BOSTROM, 2005, p. 7, tradução nossa)37. E atribui vantagens em se tornar um upload, como "[...] a capacidade de fazer cópias de segurança de si mesmo [...] e a capacidade de transmitir a si mesmo, como informação na velocidade da luz" (BOSTROM, 2005, p. 7, tradução nossa) ${ }^{3}$. Além da possibilidade de viver "[...] na realidade virtual ou diretamente na realidade física, controlando um proxy de robô" (BOSTROM, 2005, p. 7, tradução nossa)39.

Outro tópico abordado pelo filósofo sueco é a respeito da possibilidade de ir além da limitação sensorial que integra a natureza humana atualmente. As modalidades sensoriais humanas, segundo ele, poderiam se desenvolver muito mais e serem mais potencializadas (BOSTROM, 2005). O próprio conhecimento acerca das capacidades de outros animais e seus atributos possibilitam refletir acerca das mais variadas formas de interações possíveis que os seres vivos estabelecem com a natureza. Alguns deles possuem "[...] sonar, orientação magnética ou sensores de eletricidade e vibração; muitos têm um senso muito mais profundo de cheiro, visão mais nítida, etc" (BOSTROM, 2005, p. 7, tradução nossa)40. O autor reforça ainda que “[...] não há razão para pensar que o que temos esgota o alcance do possível, e certamente podemos imaginar níveis mais altos de sensibilidade e capacidade de resposta" (BOSTROM, 2005, p. 7, tradução nossa) ${ }^{41}$.

O fator limitador da morte é também lembrado por Bostrom. O filósofo argumenta que "Por causa das condições precárias em que nossos ancestrais do Pleistoceno viveram, o tempo de vida humana evoluiu para um insignificante sete

\footnotetext{
35 We enhance our natural immune systems by getting vaccinations, and we can imagine further enhancements to our bodies that would protect us from disease or help us shape our bodies according to our desires.

${ }^{36}$ Expressão característica da área de simulação computacional que significa: "em ou através de uma simulação computacional".

$37[\ldots .$.$] replicating in silico the detailed computational processes that would normally take place in a$ particular human brain.

${ }^{38}[. .$.$] the ability to make back-up copies of oneself [...] and the ability to transmit oneself as$ information at the speed of light.

$39[\ldots]$ virtual reality or directly in physicalreality by controlling a robot proxy.

$40[\ldots]$ sonar, magnetic orientation, or sensors for electricity and vibration; many have a much keener sense of smell, sharper eyesight, etc.

${ }^{41}[\ldots]$ there is no reason to think that what we have exhausts the range of the possible, and we can certainly imagine higher levels of sensitivity and responsiveness.
} 
ou oito décadas. Este é, de muitas perspectivas, um período bastante curto." (BOSTROM, 2005, p. 5, tradução nossa) ${ }^{42}$. E ainda provoca reflexões ao elucubrar sobre como teria sido a vida de grandes gênios como Beethoven ou Goethe, se estivessem ainda vivos e pudessem usufruir de uma vida saudável e cheia de vitalidade. Talvez, "[...] teriam continuado a crescer como homens e artistas, até atingir níveis de maturidade que mal podemos imaginar" (BOSTROM, 2005, p. 6, tradução nossa) 43 .

O autor discorre acerca de outro aspecto constitutivo, porém limitante, da natureza humana: os nossos humores, energia e autocontrole. Devido a sua constituição físico-biológica limitadora, o ser humano fica "[...] à mercê de um setpoint geneticamente determinado para nossos níveis de bem-estar, somos limitados em relação à energia, força de vontade e capacidade de moldar nosso próprio caráter de acordo com nossos ideais” (BOSTROM, 2005, p. 7, tradução nossa)44. O filósofo esclarece a questão ao apresentar a seguinte afirmação:

\begin{abstract}
Mesmo certos objetivos "simples", como perder peso ou parar de fumar, provam-se inatingíveis para muitos. Algum subconjunto desses tipos de problemas pode ser necessário, em vez de depender de nossa natureza atual. Por exemplo, não podemos simultaneamente ter a capacidade de romper facilmente qualquer hábito e a capacidade de formar hábitos estáveis e difíceis de romper. (Nesse sentido, o melhor que se pode esperar pode ser a capacidade de se livrar facilmente dos hábitos que não escolhemos deliberadamente para nós mesmos, e talvez um sistema de formação de hábitos mais versátil que nos permita escolher com mais precisão quando adquirir um hábito e quanto esforço deve custar para rompê-lo) (BOSTROM, 2005, p. 7, tradução nossa)45.
\end{abstract}

O filósofo observa que as limitações que constituem o ser humano e definem seu estado de natureza acabam por impedir inéditas experimentações em sua

\footnotetext{
${ }^{42}$ Because of the precarious conditions in which our Pleistocene ancestors lived, the human lifespan has evolved to be a paltry seven or eight decades. This is, from many perspectives, a rather short period of time.

$43[\ldots]$ they would have continued to grow as men and artists, to reach levels of maturity that we can barely imagine.

$44[\ldots]$ at the mercy of a genetically determined setpoint for our levels of well-being, we are limited in regard to energy, will-power, and ability to shape our own character in accordance with our ideals. 45 Even such "simple" goals as losing weight or quitting smoking prove unattainable to many. Some subset of these kinds of problems might be necessary rather than contingent upon our current nature. For example, we cannot both have the ability easily to break any habit and the ability to form stable, hard-to-break habits. (In this regard, the best one can hope for may be the ability to easily get rid of habits we didn't deliberately choose for ourselves in the first place, and perhaps a more versatile habit-formation system that would let us choose with more precision when to acquire a habit and how much effort it should cost to break it).
} 
relação com a realidade. O ser humano se encontra num estado de cognição que é determinado por todo o processo de evolução natural e em algum aspecto pela cultura e desenvolvimento da linguagem. Contudo, as limitações cognitivas que determinam o estado atual do ser humano são encaradas pela perspectiva transumanista como algo que deve ser superado, de forma a aprimorar o estado cognitivo atual caracterizado como um dos aspectos determinantes da natureza humana. A respeito dessas limitações que abrangem o aspecto cognitivo do ser humano, expõe Bostrom:

\begin{abstract}
A impossibilidade a que me refiro é mais parecida com a impossibilidade de nós, os humanos atuais, visualizarem uma hiper-esfera de 200 dimensões ou lerem, com perfeita recordação e compreensão, todos os livros da Biblioteca do Congresso. Essas coisas são impossíveis para nós porque, em poucas palavras, nos falta a capacidade cerebral. [...] Além disso, nossos cérebros humanos podem limitar nossa capacidade de descobrir verdades filosóficas e científicas. É possível que o fracasso da pesquisa filosófica em obter respostas sólidas e geralmente aceitas para muitas das grandes questões filosóficas tradicionais se deva ao fato de não sermos suficientemente inteligentes para ter sucesso nesse tipo de investigação. Nossas limitações cognitivas talvez estejam confinando-nos em uma caverna platônica, onde o melhor que podemos fazer é teorizar sobre "sombras", isto é, representações que são suficientemente simplificadas demais e embaçadas para caber dentro do cérebro humano (BOSTROM, 2005, p. 6, tradução nossa) ${ }^{46}$.
\end{abstract}

Portanto, fica evidenciado que na perspectiva transumanista a natureza humana não é concebida como algo estático, permanente ou imutável, mas sim, dotada da capacidade de se modificar, o que é amplamente evidenciado pela história evolutiva do ser humano. Por conta disso, segundo o projeto transumanista os meios que a humanidade dispõe para a modificação e melhoramento daquilo que constitui atualmente o homem devem ser encorajados de forma a proporcionar melhorias em todas as esferas da condição humana. Nesse sentido é que o melhoramento cognitivo, como outros tipos de melhoramento, não deve ser encarado como empecilho pelo fato de supostamente atentar contra uma natureza humana, pois como já foi exposto, tal natureza não é concebida em termos

${ }^{46}$ The impossibility that I am referring to is more like the impossibility for us current humans to visualize an 200-dimensional hypersphere or to read, with perfect recollection and understanding, every book in the Library of Congress. These things are impossible for us because, simply put, we lack the brainpower. [...] Further, our human brains may cap our ability to discover philosophical and scientific truths. It is possible that failure of philosophical research to arrive at solid, generally accepted answers to many of the traditional big philosophical questions could be due to the fact that we are not smart enough to be successful in this kind of enquiry. Our cognitive limitations may be confining us in a Platonic cave, where the best we can do is theorize about "shadows", that is, representations that are sufficiently oversimplified and dumbed-down to fit inside a human brain. 
essencialistas. Além disso, os transumanistas afirmam o quão impossibilitado é o ser humano em sua constituição físico-biológica atual, e que os melhoramentos oriundos da convergência tecnológica acabariam por proporcionar uma extrapolação positiva dessas limitações propiciando experiências inéditas na esfera epistemológica, sensitiva, cognitiva, etc.

\section{Considerações finais}

Ao longo deste trabalho se buscou apresentar como o melhoramento cognitivo se caracteriza como parte do projeto de melhoramento humano visado pelo transumanismo. Podemos identificar alguns tipos de limitações da condição humana que o projeto transumanista almeja amplificar, melhorar ou aprimorar. A limitação cognitiva é uma delas. A condição cognitiva partilhada pelos seres humanos atualmente impossibilita a realização de inéditas experiências a nível epistemológico na relação entre o ser humano e a realidade, além de impedir profundos resultados do trabalho cognitivo. Mentes brilhantes, quando surgem, sempre tendem a ser minoria.

A literatura transumanista tende apresentar o melhoramento cognitivo como algo que sempre fez parte da história humana, como por exemplo a revolução cognitiva operada pela linguagem e a educação. Além disso, pode-se encontrar outros exemplos de melhoramento cognitivo, tais como o uso de recursos naturais que melhoram o desempenho cognitivo com substâncias estimulantes como o café ou a nicotina. A biotecnologia farmacêutica com as substâncias nootrópicas auxiliam quimicamente no processo de cognição, ao proporcionar aumento da capacidade cognitiva, como por exemplo a concentração (uso da Ritalina), ou o melhor desempenho da memória. Outra proposta de melhoramento cognitivo é a Estimulação Magnética Transcraniana (EMT), que estimula os neurônios no córtex cerebral, alterando o campo magnético induzido por uma bobina presa à cabeça. Pode aumentar ou diminuir a excitabilidade do córtex, alterando assim seu nível de plasticidade. Também se evidenciou a vasta gama de tecnologia da informação que possuem um papel de melhoramento cognitivo, tais como as tecnologias que ligam o ser humano a hardwares externo de forma a propiciar uma estensão da cognição. O melhoramento coletivo seria um tipo de melhoramento que operaria de forma a aumentar o desempenho dos membros individuais do grupo e por 
consequência também coletivo, exemplos disso seriam a Internet e o e-mail, que podem ser considerados como grandes softwares de aprimoramento cognitivo. Por fim, a manipulação genética (ou a seleção genética), que fornece aparato mais eficaz do que a psicofarmacologia, já que visa modificar de forma permanente desde o "fundamento" o ser humano, além de que a modificação genética tornaria o indivíduo independente de um suprimento externo, como um medicamento.

Ao longo do artigo foram expostos também alguns exemplos de limitações derivadas da condição biológica humana que o transumanismo visa superar. A limitação sensorial é a limitação que impossibilita o desenvolvimento das capacidades sensoriais de serem potencializadas. Certos animais detêm capacidades sensoriais que o humano não detém, como por exemplo, a propriedade do sonar, a orientação magnética, sensores de eletricidade e vibração. Esses exemplos servem para mostrar que existem possibilidades a serem desbravadas neste campo.

Outro aspecto constitutivo da natureza humana, que pode ter seu limite expandido é o corpo e sua funcionalidade. O humano sempre desejou superar os limites impostos pelo seu organismo, tais como voar, curar as doenças que naturalmente adquirimos, etc. Com o desenvolvimento tecnológico a limitação corporal ganhou outro status. Apresenta-se a possibilidade de num futuro não tão distante uma interação homem-máquina, mente e computador. Neste contexto poder-se-ia romper com a limitação imposta pela natureza sobre o corpo e suas funcionalidades concebendo o ser humano numa nova forma de ser, emergido em novas experiências existenciais.

O limite da morte, do tempo de vida útil do ser humano, é outra limitação que atualmente (e desde há muito tempo) tenta-se extirpar, ou ao menos prolongar, proporcionando aos grandes gênios maior tempo de contribuição de seu trabalho para a humanidade. Claro que este tipo de limite expandido (como os outros) demanda muitas reflexões sobre os impactos na vida social.

Por fim, outro aspecto limitante constitutivo da natureza humana são os nossos humores, energia e autocontrole. Nesse sentido, acabamos por sucumbir a muitos desejos e vontades por estarmos condicionados pela nossa estrutura constitutiva físico-biológica. Um exemplo disso seria o desejo de parar de fumar, que muitos não concretizam devido ao fato de estarem dependentes da nicotina. Isso demostra o quão vulnerável o ser humano pode estar diante de forças viciosas 
acarretadas por substâncias específicas.

A proposta transumanista do melhoramento cognitivo desemboca em muitas questões e problemas de ordem filosófica, dentre eles a questão voltada à natureza humana. Para o transumanismo de uma forma geral, a natureza humana é entendida como algo em constante mutação, aos moldes do pantha rei heraclítico, porém aqui entendida como algo que está sempre a vir-a-ser e que por isso não deve temer o controle e domínio do próprio homem sobre como virá a ser este vir-aser. Portanto, as mudanças que visam promover a melhoria da qualidade de vida e da produção humana (em todas as esferas da realidade), devem ser incentivas. É claro que o debate filosófico se torna indispensável, pois é justamente ele que pensará sobre as implicações dessas melhorias, se tal melhoria é de fato uma melhoria, e assim por diante.

\section{Referências}

BOSTROM, Nick. Human Genetic Enhancements: A Transhumanist Perspective. The Journal of Value Inquiry, v.37, n. 4, p. 493-506, $2003 \mathrm{a}$.

BOSTROM, Nick. Transhumanist Values. In: ADAMS, Frederick. Ethical Issues for the 21st Century, v. 30, Issue Supplement, p. 3-14, 2005.

BOSTROM, Nick. Superinteligência: Caminhos, perigos, estratégias. São Paulo. Darkside, 2018.

BOSTROM, Nick; SANDBERG, Anders. Converging Cognitive Enhancements. Ann. N.Y. Acad. Sci. v. 1093, p. 201-227, 2006.

BOSTROM, Nick; SAVULESCU, Julian. Human enhancement ethics: The state of the debate. In: SAVULESCU, Julian; BOSTROM, Nick. (Eds.). Human enhancement. Oxford: Oxford University Press, 2009, p. 1-25.

BUCHANAN, Allen. Human nature and enhancement. Bioethics, v. 23, n. 3, p. 141150, 2009.

DANIELS, Norman. Can anyone really be talking about ethically modifying human nature? In: SAVULESCU, J.; BOSTROM, Nick. (Eds.). Human enhancement. Oxford: Oxford University Press, 2009, p. 25-42.

FERRY, Luc. A revolução transhumanista. São Paulo: Manoele, 2018.

NIELSEN, Lisbeth Witthøfft. The Concept of Nature and the Enhancement Technologies Debate. In: SAVULESCU, Julian; MEULEN, Ruud ter; KAHANE, 
Guy (Org.). Enhancing Human Capacities. New Jersy: Wiley-Blackwell, 2011, p. 19-33.

SANDBERG, Anders. Cognition enhancement: Upgrading the brain. In:

SAVULESCU, Julian; MEULEN, Ruud ter; KAHANE, Guy (Org.). Enhancing Human Capacities. Wiley-Blackwell, New Jersey: 2011, p. 71-91.

VILAÇA, Murilo Mariano; DIAS, Maria Clara Marques. Transumanismo e o futuro (pós) humano. Physis Revista de Saúde Coletiva, Rio de Janeiro, v. 24, n.2, p. 341-362, 2014.

Recebido em: 15/07/2021. Aprovado em: 06/10/2021. Publicado em: 27/12/2021. 